\title{
Characterization of Semilumped CPW Elements for Millimeter-Wave Filter Design
}

\author{
Farshid Aryanfar, Student Member, IEEE, and Kamal Sarabandi, Fellow, IEEE
}

\begin{abstract}
In this paper, two accurate models for interdigital capacitors and shunt inductive stubs in coplanar-waveguide structures are presented and validated over the entire $W$-band frequency range. Using these models, a novel bandpass filter (BPF) and a miniaturized high-pass filter are designed and fabricated. By inserting interdigital capacitors in BPF resonators, an out-of-band transmission null is introduced, which improves rejection level up to $17 \mathrm{~dB}$ over standard designs of similar filters. A high-pass filter is also designed, using semilumped-element models in order to miniaturize the filter structure. It is shown that a fifth-order high-pass filter can be built with a maximum dimension of less than $\lambda_{g} / 3$. Great agreement between simulated and measured responses of these filters is demonstrated.
\end{abstract}

Index Terms-Bandpass, coplanar waveguide (CPW), filter, high-pass, interdigital capacitor, millimeter wave, semilumped, $W$-band.

\section{INTRODUCTION}

$\mathbf{M}$ ICROWAVE filters have been studied extensively using both lumped and distributed elements. However, the literature concerning planar millimeter-wave filters, especially at $W$-band frequencies, is scarce. There are few difficulties involved in filter design and fabrication at millimeter-wave frequencies and above. Most parasitic elements can usually be ignored at lower frequencies. However, their effects become significant at millimeter-wave frequencies. The parasitic elements and their effects cannot usually be considered as design parameters. Hence, they have to be accurately modeled and compensated for. To avoid dealing with the parasitic effects, use of structures with minimal parasitic features can be considered. Coplanar waveguide (CPW) line discontinuities are well characterized at microwave frequencies [1]-[3] and have been studied to some extent at higher frequencies up to $50 \mathrm{GHz}$ [4]-[6]. However, modeling and characterization of such discontinuities at $W$-band frequencies is rather limited. Calibration accuracy at the $W$-band is one of the major difficulties in characterizing parasitic capacitances and inductances, which can be as small as a fraction of a femtofarad and a picohenry, respectively. Another problem is that the dimensions of typical lumped elements become comparable with the wavelength and require more complicated models to achieve reasonable accuracy. This paper provides accurate models for semilumped elements, which facilitate a systematic approach for filter design at millimeter waves.

Manuscript received May 24, 2004; revised July 27, 2004. This work was supported by the U.S. Army Research Office.

The authors are with the Radiation Laboratory, Department of Electrical Engineering and Computer Science, The University of Michigan at Ann Arbor, Ann Arbor, MI 48109 USA (e-mail: faryanfa@umich.edu; saraband@eecs.umich.edu).

Digital Object Identifier 10.1109/TMTT.2005.845748

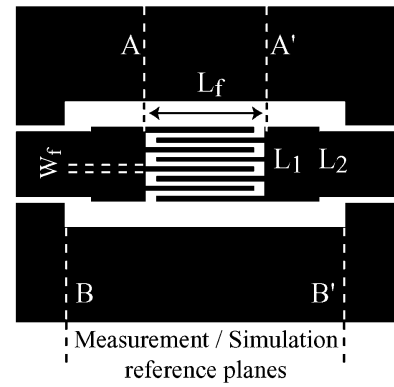

(a)

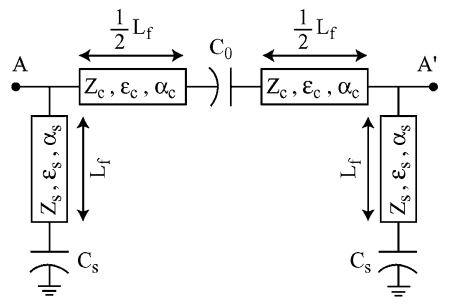

(b)
Fig. 1. Interdigital capacitor. (a) Layout. (b) Circuit model.

TABLE I

CPW LINE AND INTERDIGITAL CAPACITOR DIMENSIONS

\begin{tabular}{cccc}
\hline$W_{\text {Line }}$ & $G_{\text {Line }}$ & $W_{\text {Finger }}$ & $G_{\text {Finger }}$ \\
\hline $115 \mu \mathrm{m}$ & $40 \mu \mathrm{m}$ & $7.5 \mu \mathrm{m}$ & $7.5 \mu \mathrm{m}$ \\
\hline
\end{tabular}

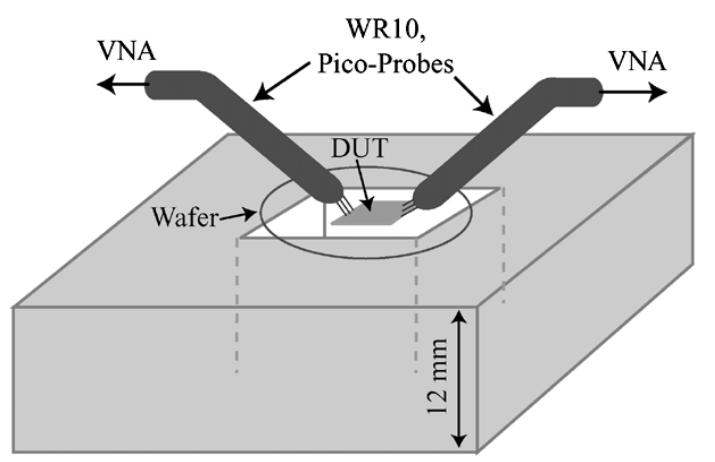

Fig. 2. Wafer holder with a cavity under the device-under-test (DUT).

TABLE II

MODEl PARAMETERS FOR INTERDIGITAL CAPACITOR

\begin{tabular}{cccccc}
\hline$Z_{c}(\Omega)$ & $Z_{s}(k \Omega)$ & $\varepsilon_{c}$ & $\varepsilon_{s}$ & $\alpha_{c}(d B / c m)$ & $\alpha_{s}(d B / c m)$ \\
\hline 96 & 5.4 & 2.5 & 3.4 & 2.5 & 3.7 \\
\hline
\end{tabular}

Furthermore using a parasitic component of the proposed model as a design parameter, an out-of-band transmission null in a bandpass-filter (BPF) response is introduced.

In Section II, an accurate model for series interdigital capacitors in CPW lines is introduced and validated over a wide range of physical dimensions. In Section III, an existing model for shunt inductive stubs [7] is then modified to be valid at $W$-band frequencies. In Sections IV and V, design, fabrication, and measurements of a novel BPF and a miniaturized high-pass filter are presented, which make use of the semilumped elements. 


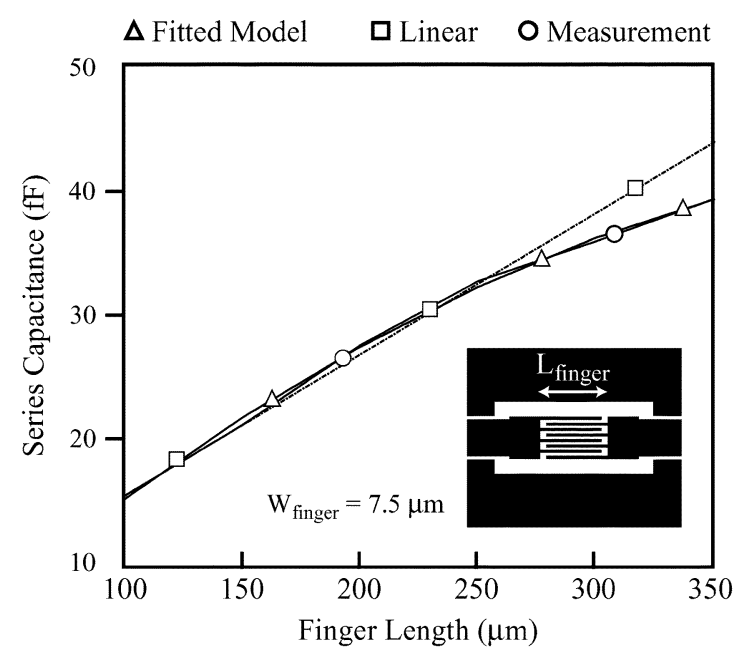

Fig. 3. Series capacitance of interdigital capacitors in CPW lines.

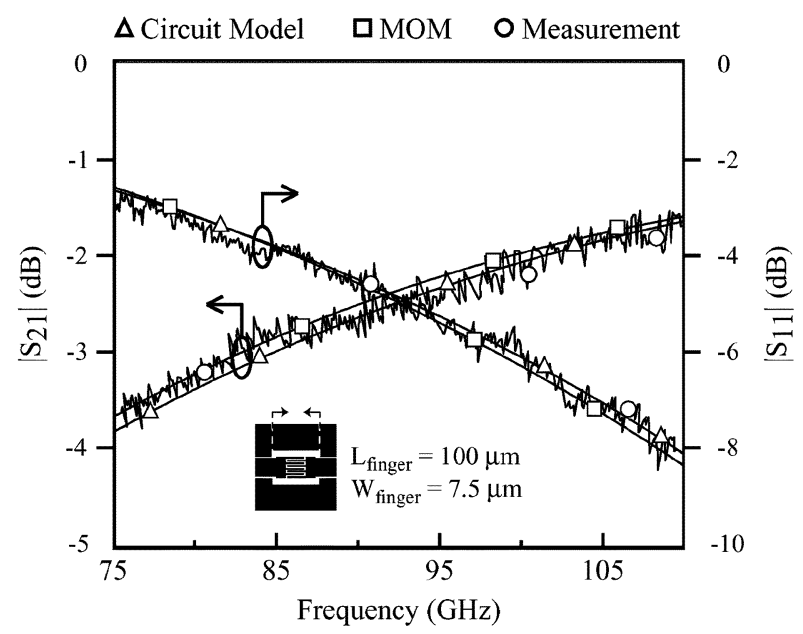

(a)

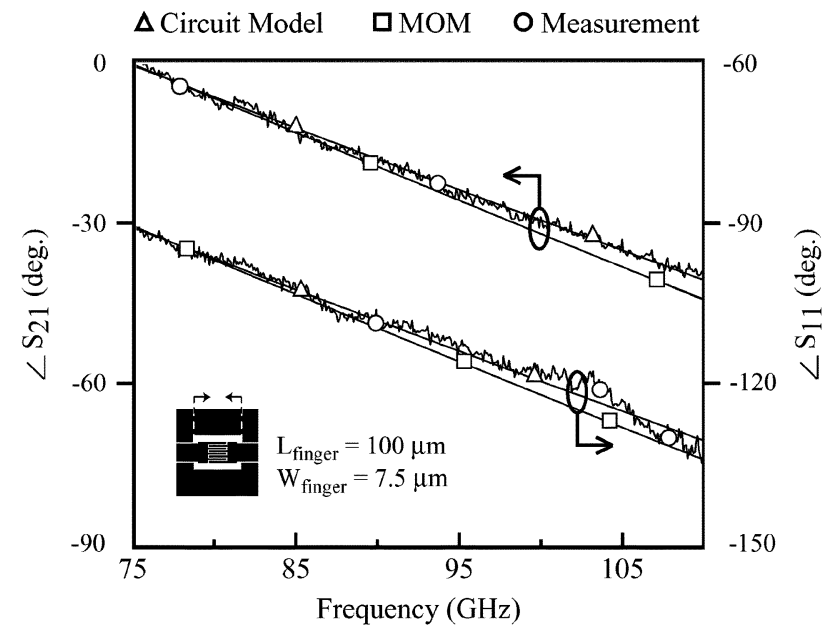

(b)

Fig. 4. Measured and simulated $S$-parameters of interdigital capacitor $L_{f}=$ $100 \mu \mathrm{m}$. (a) Magnitude. (b) Phase.

\section{INTERDIGITAL CAPACITORS IN CPW LINES}

Interdigital capacitors are used either far below their resonant frequencies [8]-[11] or as quarter-wavelength series open stubs [2], [6] to ensure the accuracy of simple existing models

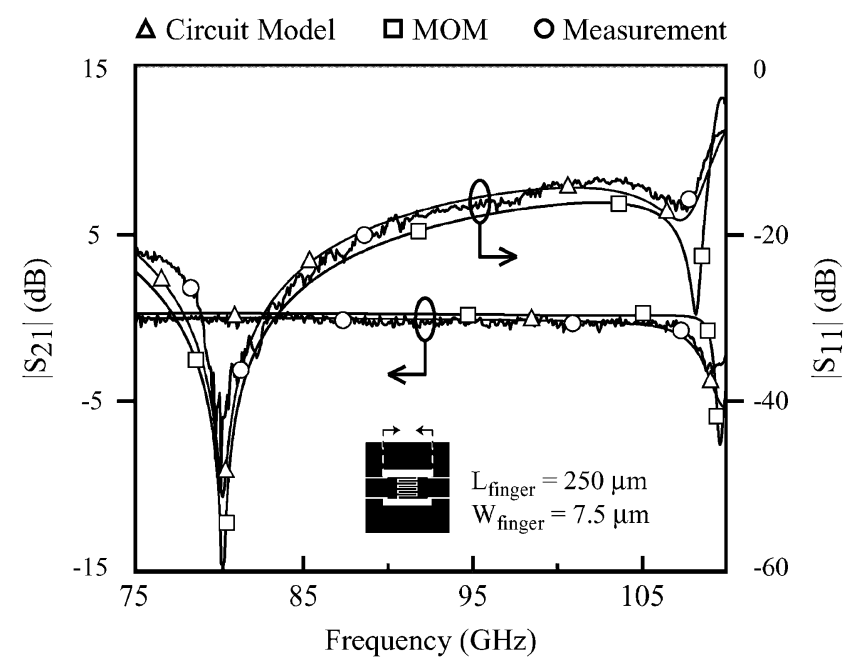

(a)

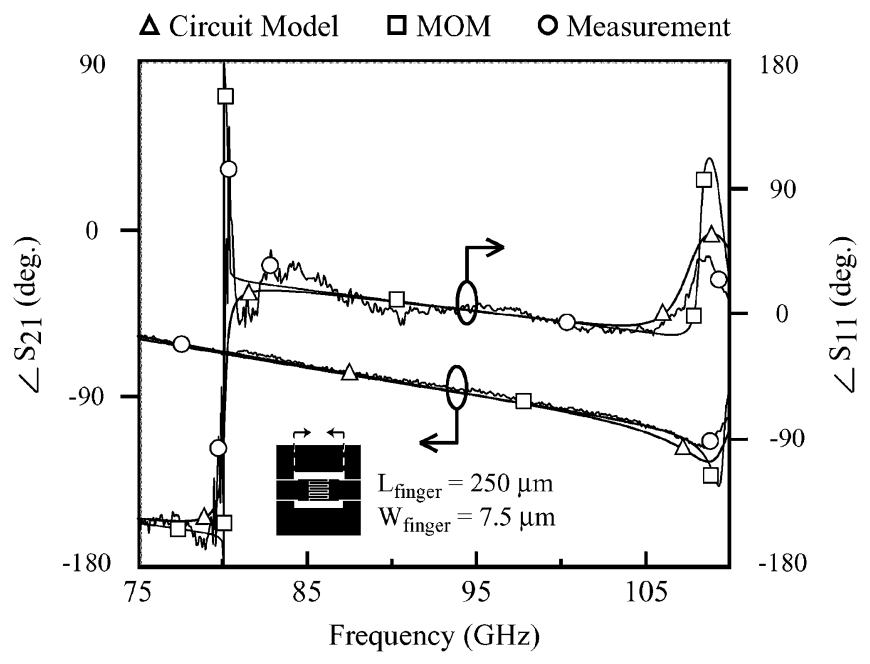

(b)

Fig. 5. Measured and simulated $S$-parameters of interdigital capacitor $L_{f}=$ $250 \mu \mathrm{m}$. (a) Magnitude. (b) Phase.

(lumped capacitor and open stub, respectively). For the quarterwavelength open-stub case, complicated models for capturing the behavior of parasitic elements have been considered to increase the accuracy of the stub model [1], [12]. However, the large number of parameters introduced in these models limits their applicability. Despite the complexity, the accuracy of these models is still insufficient for applications in $W$-band frequencies.

Due to limitations in width of the center conductor of CPW lines (for preventing transversal mode radiation) and minimum achievable gap size (limited by the fabrication process), large capacitances can only be achieved by increasing capacitor finger length. Therefore, a complete model, which can accurately represent interdigital capacitor behavior over a wide range of finger lengths, and with a minimum number of parameters, is needed to facilitate design procedure.

Here, a new physics-based model is introduced and validated over the entire $W$-band frequency range for different capacitor finger length values. Fig. 1(a) and (b) shows the layout and circuit model of the interdigital capacitor, respectively. In order to extract the capacitor model, the effect of extra lines between $A$ 


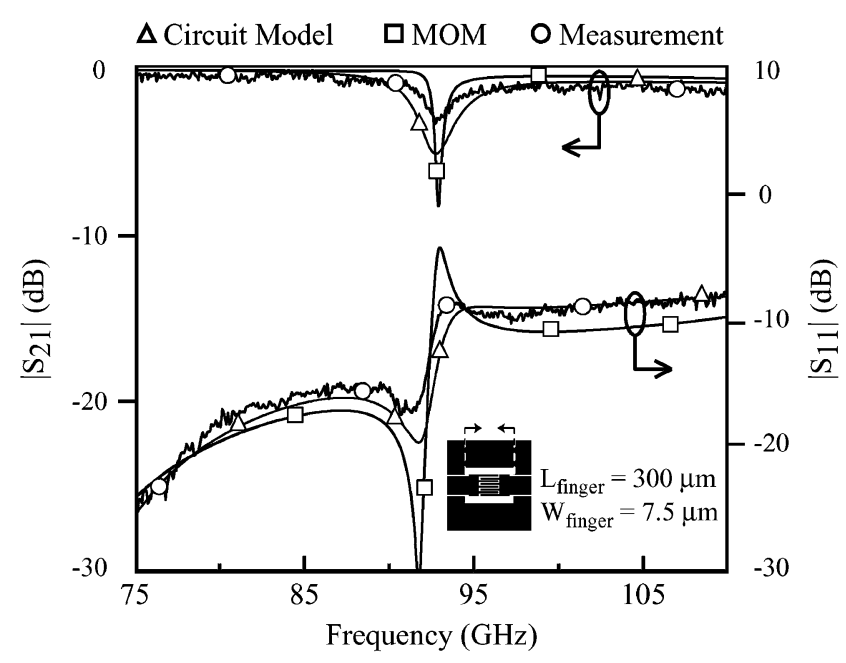

(a)

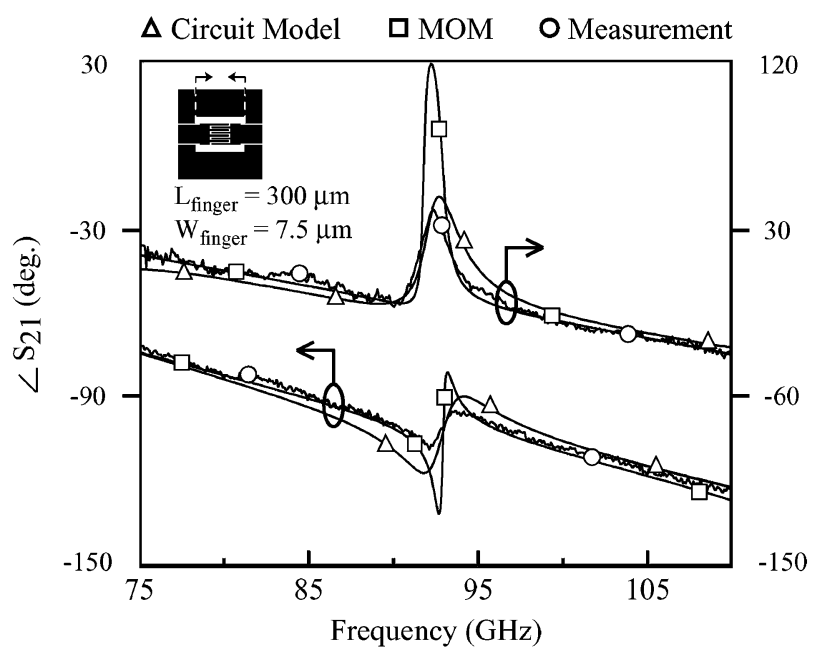

(b)

Fig. 6. Measured and simulated $S$-parameters of interdigital capacitor $L_{f}=$ $300 \mu \mathrm{m}$. (a) Magnitude. (b) Phase.

and $B$, as well as $A^{\prime}$ and $B^{\prime}$, are deembedded. The discontinuity between lines $L_{1}$ and $L_{2}$ is negligible because the impedance change is only approximately $3 \%$. For a fixed CPW line geometry, finger width $\left(W_{f}\right)$, which is selected to be equal to gap width, determines the effective dielectric constants $\left(\varepsilon_{c}, \varepsilon_{s}\right)$, the attenuation constants $\left(\alpha_{c}, \alpha_{s}\right)$, and the characteristic impedances $\left(Z_{c}, Z_{s}\right)$ of the series and shunt line segments of the circuit model. $C_{0}$ and $C_{s}$ are functions of finger length $\left(L_{f}\right)$ only.

The dimensions of a typical CPW line and interdigital capacitor finger length and gap width are given in Table I. The capacitor shown in Fig. 1(a) has been fabricated with different lengths ranging from 100 to $400 \mu \mathrm{m}$ on a 10-mil-thick quartz wafer. The $S$-parameter measurements for these capacitors in $W$-band $(75-110 \mathrm{GHz}$ ) were realized using a probe station (for on-wafer measurements), HP-8510C network analyzer, and HP-W85104A millimeter-wave test setup.

The probe station chuck, which holds the wafers, changes a CPW line to a conductor-backed CPW line and, thus, it affects the phase constant of the line [13] and the values of the parasitic elements. In order to eliminate these effects, a supporting structure with a cavity in the middle (shown in Fig. 2) is built and

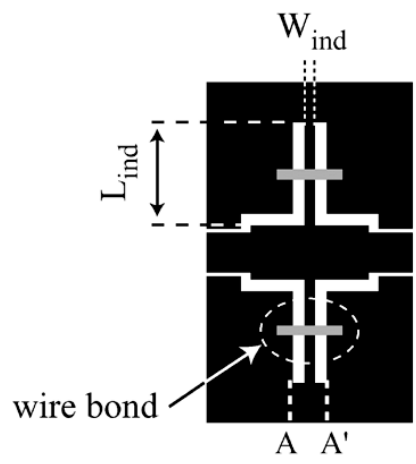

(a)

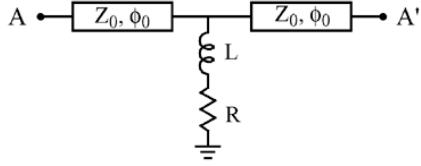

(b)
Fig. 7. Characterization of effective inductance and resistance for short stubs in CPW line. (a) Inductor layout. (b) Circuit model.

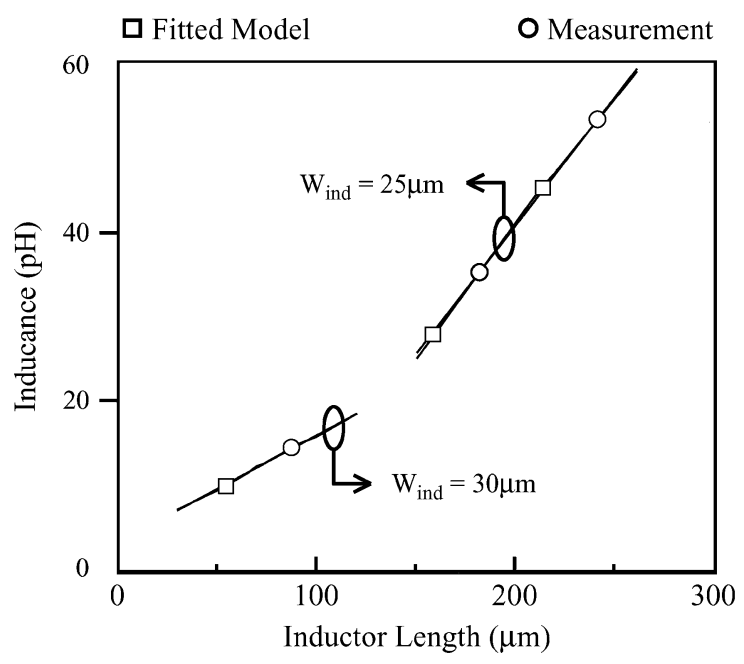

Fig. 8. Inductance of short-circuit stubs in CPW lines.

placed on top of the probe station's chuck. Method of moments (MOM) and circuit simulations are performed using the Agilent Advanced Design System (ADS). For the dimensions given in Table I, the effective dielectric constants, attenuation constants, and characteristic impedances of the circuit model are extracted by comparing the measured and simulated $S$-parameters (magnitude and phase) for 11 different cases $(100-350 \mu \mathrm{m})$. These are provided in Table II. The values of $C_{0}$ and $C_{s}$ are only dependent on normalized finger length $\left(l_{f}=L_{f} / L_{f 0}\right)$ and are given by

$$
\begin{aligned}
& C_{0}=C_{00}\left(-0.12 l_{f}^{2}+1.19 l_{f}-0.09\right) \\
& C_{s}=C_{s 0}\left(0.6+0.4 l_{f}\right)
\end{aligned}
$$

where $C_{00}=15.9 \mathrm{fF}$ and $C_{s 0}=0.1 \mathrm{fF}$ are values of $C_{0}$ and $C_{s}$ at $L_{f 0}=100 \mu \mathrm{m}$, respectively. The relation between the capacitance value and its physical length for short lengths is following a linear behavior, hence, these equations are expected to also be valid for length values smaller than $100 \mu \mathrm{m}$. The maximum error is less than $4 \%$ over the mentioned range.

Fig. 3 compares the series capacitance $\left(C_{0}\right)$ value, given by (1), with its measured value. As shown, for finger-length values above $250 \mu \mathrm{m}$, capacitance does not increase linearly with finger length. To capture capacitance behavior for higher length 


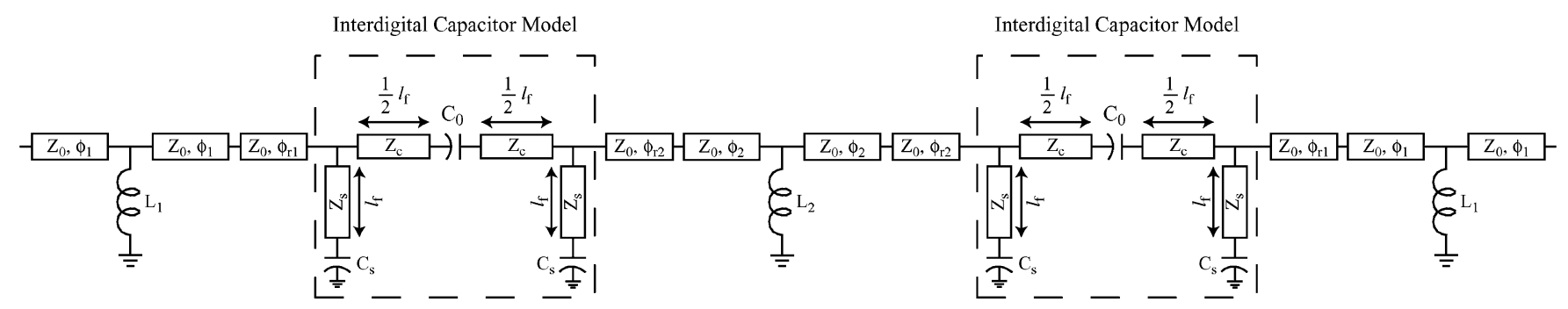

Fig. 9. New inductive coupled-resonator BPF circuit model.

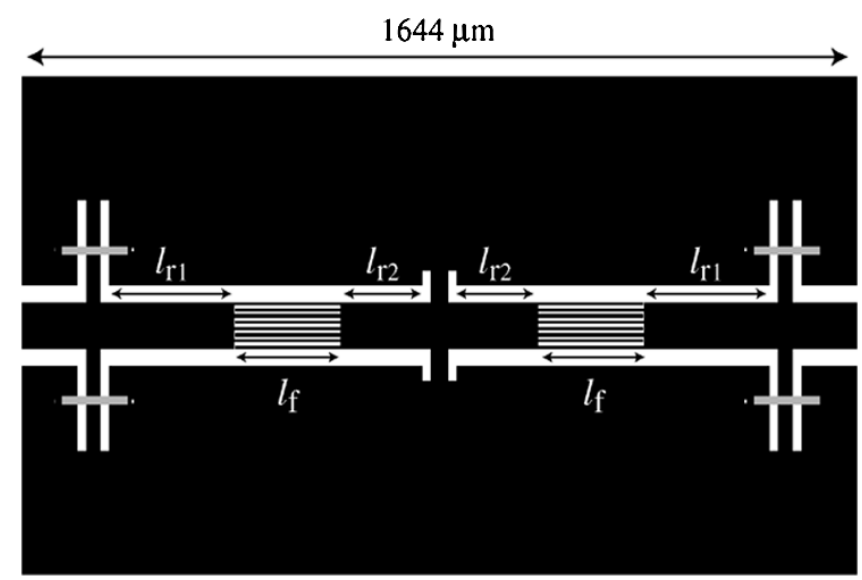

Fig. 10. New inductive coupled-resonator BPF layout.

values, a second-order term is provided in (1). Figs. 4-6 compare the measured $S$-parameters (magnitude and phase) with the MOM and circuit model simulation results for three different capacitor finger-length values of 100, 250, and $300 \mu \mathrm{m}$. The circuit model results show excellent agreement with the measurements in all situations. The circuit results show an even better agreement with the measurement than the MOM result. The reason is that MOM simulations in ADS for CPW structures are based on magnetic current modeling and, thus, cannot handle metallic losses.

\section{SHUNT INDUCTORS IN CPW LINES}

Here, short-circuit stubs, shown in Fig. 7(a), which are often used as inverters in filter designs [7], [14] are characterized at $W$-band for different stub length and width values. As the accuracy of the closed-form equation for inductance [7] is quite poor at $W$-band frequencies (errors often greater than 100\%), inductance values have been extracted by comparison between simulation results for the circuit model shown in Fig. 7(b) and measured data for 20 different cases. The inductance values versus inductor lengths for two different inductor widths are shown in Fig. 8. As demonstrated, the inductance has a linear relation with the stub length within the selected range. This relationship can be described by

$$
L_{\text {ind }}(\mathrm{pH})= \begin{cases}L_{w 1}\left(0.49 l_{1}+0.5\right) & W_{\text {ind }}=30 \mu \mathrm{m} \\ L_{w 2}\left(1.76 l_{2}-0.75\right) & W_{\text {ind }}=25 \mu \mathrm{m}\end{cases}
$$

where $L_{w 1}=7.7 \mathrm{pH}$ and $L_{w 2}=25.4 \mathrm{pH}$ are inductance values at $l_{10}=30 \mu \mathrm{m}$ and $l_{20}=150 \mu \mathrm{m}$, respectively, and $l_{1}=$ $L_{\text {ind }} / l_{10}, l_{2}=L_{\text {ind }} / l_{20}$.

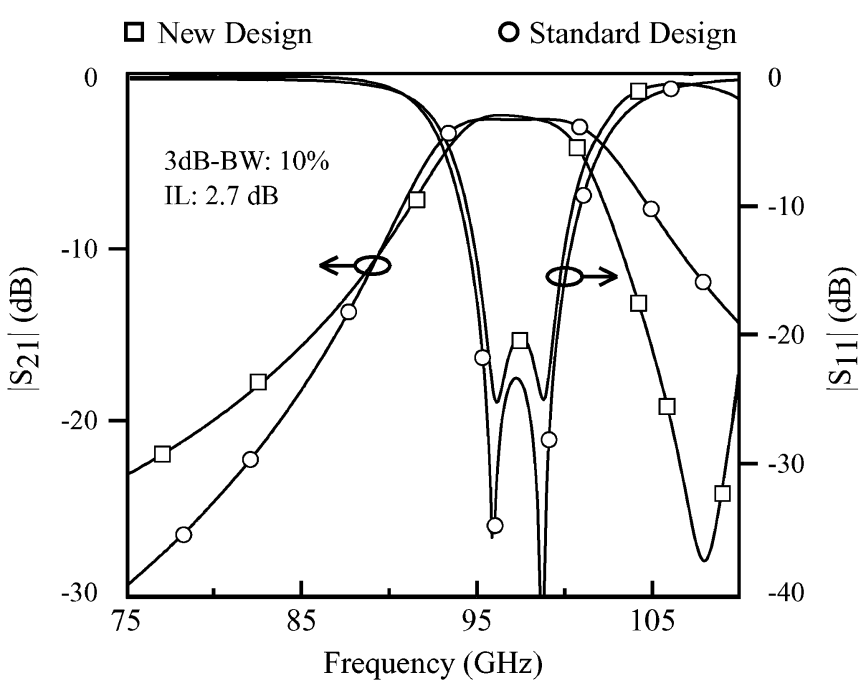

Fig. 11. Simulation results for the new inductive coupled-resonator BPF versus standard type of this filter.

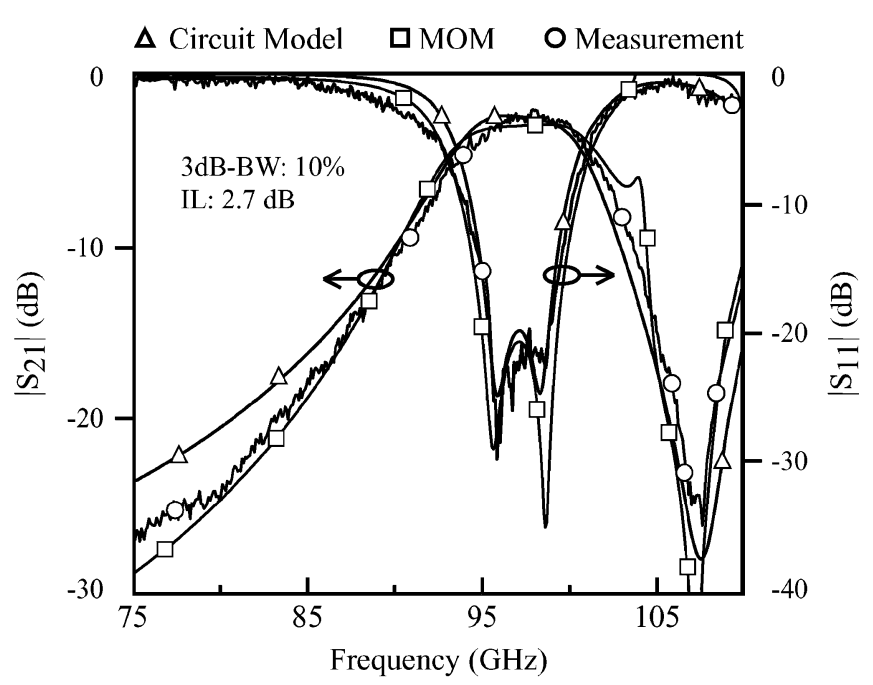

Fig. 12. Simulation and measurement results for the new inductive coupled-resonator BPF.

\section{BPF}

Here, we demonstrate the usefulness of the accurate models of the semilumped elements introduced in the previous sections. A standard inductive coupled-resonator BPF [7] is modified by placing an interdigital capacitor in each resonators section, as shown in Figs. 9 and 10. The equivalent shunt loaded stubs of the interdigital capacitors [see Fig. 1(b)] provide a transmission 


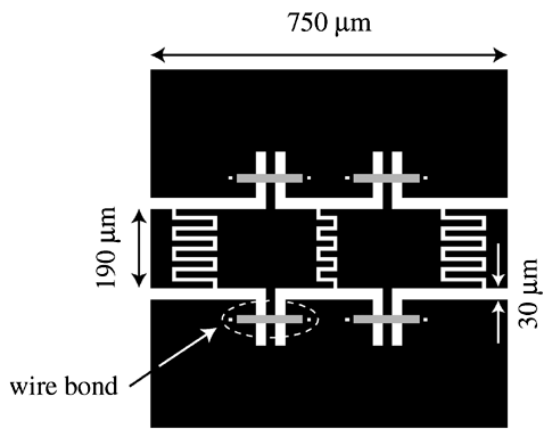

Fig. 13. Miniaturized high-pass filter layout.

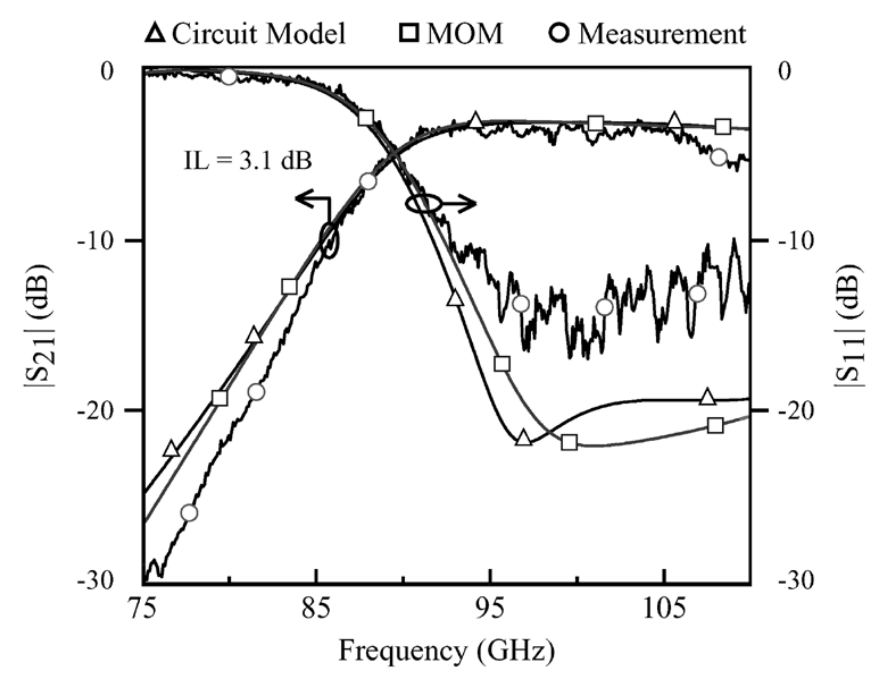

Fig. 14. Simulation and measurement results for the miniaturized high-pass filter.

null, and this is used to improve the out-of-band rejection response. The position of the null can be controlled simply by the length of the interdigital capacitor fingers. Fig. 11 compares simulation results for the new filter with a standard one. As shown, the $3-\mathrm{dB}$ bandwidth of the new filter is slightly $(10 \%)$ less than the standard filter, while the rejection at a desired frequency can be improved by $20 \mathrm{~dB}$. Due to minor radiation loss from the interdigital capacitors, the quality factor $(Q)$ of the resonators is slightly decreased in comparison with their values for the standard resonators. Consequently, the measured results of this filter shows $0.5 \mathrm{~dB}$ more insertion loss. This filter was fabricated on a 10-mil-thick quartz wafer using standard lithography and wet etching on 3- $\mu$ m electroplated gold. Fig. 12 shows good agreement between the measured and simulated results of the circuit model. In Fig. 12, the nonphysical MOM response around $104 \mathrm{GHz}$ occurs because of MOM simulator failure.

\section{Miniaturized High-PASS FiLTER}

The main goal of modeling semilumped elements is to use them as lumped elements in filter design. Lumped-element filters have very compact size, which can be beneficial in numerous applications. Using a ladder network of three series capacitors and two shunt inductors, which were modeled and described in Sections II and III, a miniaturized high-pass filter, shown in Fig. 13, is designed. As can be seen, the total length of the filter is only $750 \mu \mathrm{m}$, which is less than $\lambda_{g} / 3$ at the cutoff frequency of the filter. This filter was also fabricated on a quartz wafer, and its measured and simulated results are compared in Fig. 14. It can be seen that circuit model results have good agreement with both the MOM and measurement results. There are second-order effects such as interactions between inductors and capacitors that cannot be estimated by the circuit model, and these can be a source for degrading return loss. However, it should be noted the circuit model is still working as good as MOM.

\section{CONCLUSION}

Simple and accurate models for two semilumped CPW elements have been presented and validated at $W$-band frequencies for different dimensions. The simulation results of the extracted models show good agreement with the measured results. The models greatly simplify the process of filter design at $W$-band and higher frequencies. The semilumped elements have been used for design of two millimeter-wave filters (a BPF and high-pass filter), and have helped to improve filter response in the first case and miniaturization in the second case.

\section{REFERENCES}

[1] N. I. Dib, L. P. B. Katehi, G. E. Ponchak, and R. N. Simons, "Theoretical and experimental characterization of coplanar waveguide discontinuities for filter applications," IEEE Trans. Microw. Theory Tech., vol. 39, no. 5, pp. 873-882, May 1991.

[2] A. K. Rayit and N. J. McEwan, "Coplanar waveguide filters," in IEEE MTT-S Int. Microwave Symp. Dig., vol. 3, Jun. 1993, pp. 1317-1320.

[3] W. Menzel, W. Schwab, and G. Strauss, "Investigation of coupling structures for coplanar bandpass filters," in IEEE MTT-S Int. Microwave Symp. Dig., vol. 3, May 1995, pp. 1407-1410.

[4] S. D. Gedney, P. Petre, M. Matloubian, and R. T. Kihm, "Simulation and performance of passive millimeter wave coplanar waveguide circuit devices," in IEEE Wireless Commun. Conf., Aug. 1997, pp. 27-31.

[5] P. M. Watson and K. C. Gupta, "Design and optimization of CPW circuits using EM-ANN models for CPW components," IEEE Trans. Microw. Theory Tech., vol. 45, no. 12, pp. 2515-2523, Dec. 1997.

[6] G. E. Ponchak and L. P. B. Katehi, "Open- and short-circuit terminated series stubs in finite-width coplanar waveguide on silicon," IEEE Trans. Microw. Theory Tech., vol. 45, no. 6, pp. 970-976, Jun. 1997.

[7] J. K. A. Everard and K. K. M. Cheng, "High performance direct coupled bandpass filters on coplanar waveguide," IEEE Trans. Microw. Theory Tech., vol. 41, no. 9, pp. 1568-1571, Sep. 1993.

[8] D. F. Williams and S. E. Schwarz, "Design and performance of coplanar waveguide bandpass filters," IEEE Trans. Microw. Theory Tech., vol. 31, no. 7, pp. 558-566, Jul. 1983.

[9] K. Yoshida, K. Sashiyama, S. Nishioka, H. Shimakage, and Z. Wang, "Design and performance of miniaturized superconducting coplanar waveguide filters," IEEE Trans. Appl. Supercond., vol. 9, no. 2, pp. 3905-3908, Jun. 1999.

[10] H. Kanaya, T. Shinto, K. Yoshida, T. Uchiyama, and Z. Wang, "Miniaturized HTS coplanar waveguide bandpass filters with highly packed meander lines," IEEE Trans. Appl. Supercond., vol. 11, no. 1, pp. 481-484, Mar. 2001.

[11] Y.-K. Kuo, C.-H. Wang, and C. H. Chen, "Novel reduced-size coplanarwaveguide bandpass filters," IEEE Microw. Wireless Compon. Lett., vol. 11 , no. 2, pp. 65-67, Feb. 2001.

[12] F. L. Lin, C. W. Chiu, and R. B. Wu, "Coplanar waveguide bandpass filter-A ribbon-of-brick-wall design," IEEE Trans. Microw. Theory Tech., vol. 43, no. 7, pp. 1589-1596, Jul. 1995.

[13] D. F. Williams, "On-wafer measurement at millimeter wave frequencies," in IEEE MTT-S Int. Microwave Symp. Dig., vol. 3, Jun. 1996, pp. $1683-1686$.

[14] A. Vogt and W. Jutzi, "An HTS narrow bandwidth coplanar shunt inductively coupled microwave bandpass filter on $\mathrm{LaAlO}_{3}$," IEEE Trans. Microw. Theory Tech., vol. 45, no. 4, pp. 492-497, Apr. 1997. 


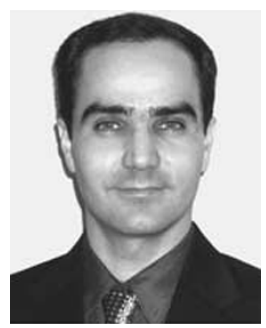

Farshid Aryanfar (S'01) was born in Mashad, Iran, in 1972. He received the B.S. degree in electrical engineering and M.S. degree with an emphasis on electromagnetics from the University of Tehran, Tehran, Iran, in 1994 and 1998, respectively, and the Ph.D. degree in electrical engineering and computer science with an emphasis on applied electromagnetics and RF circuits at The University of Michigan at Ann Arbor, in 2005. His M.S. thesis concerned wave-propagation modeling in microcellular and pico-cellular environment using the ray-tracing algorithm, which later led to a 3-D physics-based wave-propagation simulator for wireless channel characterization.

From 2000 to 2005, he was a Research Assistant with the Radiation Laboratory, The University of Michigan at Ann Arbor, where he designed and fabricated a $W$-band transceiver system for characterizing wave propagation in a scaled environment and also developed a though-wall imaging algorithm. From 1997 to 2000, he was Senior Microwave Engineer with the Informatics Services Corporation (ISC), where he was involved in developing satellite terminals and nondirectional beacon (NDB) transmitters. His current research interests include microwave/millimeter-wave circuits and systems, wave propagation in complex environment, and wireless channel characterization.

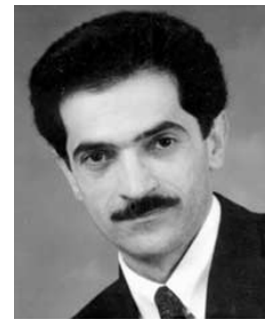

Kamal Sarabandi (S'87-M'90-SM'92-F'00) received the B.S. degree in electrical engineering from the Sharif University of Technology, Tehran, Iran, in 1980, and the M.S.E. and Ph.D. degrees from The University of Michigan at Ann Arbor, in 1986 and 1989 , respectively, both in electrical engineering.

$\mathrm{He}$ is currently the Director of The Radiation Laboratory and a Professor with the Department of Electrical Engineering and Computer Science, The University of Michigan at Ann Arbor. His research areas of interests include microwave and millimeter-wave radar remote sensing, electromagnetic-wave propagation, and antenna miniaturization. He possesses 20 years of experience with wave propagation in random media, communication channel modeling, microwave sensors, and radar systems and is leading a large research group including four research scientists and $12 \mathrm{Ph} . \mathrm{D}$. and two M.S. students. Over the past ten years, he has generated $20 \mathrm{Ph} . \mathrm{D}$. students. He was the Principal Investigator of numerous projects sponsored by the National Aeronautics and Space Administration (NASA), Jet Propulsion Laboratory (JPL), Army Research Office (ARO), Office of Naval Research (ONR), Army Research Laboratory (ARL), National Science Foundation (NSF), Defense Advanced Research Projects Agency (DARPA), and numerous industries. He has authored numerous book chapters and over 115 papers appearing in refereed journals on electromagnetic scattering, random media modeling, wave propagation, antennas, microwave-measurement techniques, radar calibration, inverse-scattering problems, and microwave sensors. He has also authored or coauthored over 230 papers and invited presentations in many national and international conferences and symposia on similar subjects. He is listed in Who's Who in American Men and Women of Science, Who's Who in America, and Who's Who in Electromagnetics.

Dr. Sarabandi is a vice president of the IEEE Geoscience and Remote Sensing Society (GRSS), chairman of the Awards Committee of the IEEE GRSS, and a member of the IEEE Technical Activities Board Awards Committee. He is the associate editor of the IEEE TRANSACTIONS ON ANTENNAS AND PROPAGATION and the IEEE SENSORS JOURNAL. He is also a member of Commission F of URSI and The Electromagnetic Academy. He was the recipient of the Henry Russel Award from the Regent of The University of Michigan (the highest honor the University of Michigan bestows on a faculty member at the assistant or associate level), the 1999 GAAC Distinguished Lecturer Award presented by the German Federal Ministry for Education, Science, and Technology, which is given to approximately ten individuals worldwide in all areas of engineering, science, medicine, and law, and a 1996 Teaching Excellence Award presented by the Electrical Engineering and Computer Science Department, The University of Michigan at Ann Arbor. Over the past several years, joint papers presented by his students at a number of symposia [IEEE Antennas and Propagation Society (IEEE AP-S) Symposia (1995, 1997, 2000, and 2001); the IEEE International Geoscience and Remote Sensing Symposium (1999); and the IEEE Microwave Theory and Techniques Society (IEEE MTT-S) International Microwave Symposium (IMS) (2001)] have been the recipients of Student Prize Paper Awards. 